\title{
Medida de energia total do leite materno de mães de trigêmeos, gêmeos e filhos únicos
}

\author{
Total energy supplied by the human milk from mothers of triplet, twin and single neonates
}

Ariane Nadólskis Severine ${ }^{1}$, Fernando José de Nóbrega², Yara Juliano ${ }^{3}$, Neil Ferreira Novo³

\section{RESUMO}

Objetivo: Comparar a energia total do leite materno de mães de trigêmeos, gêmeos e filhos únicos.

Métodos: Estudo transversal e controlado feito com mães usuárias do banco de leite do Hospital Israelita Albert Einstein. Foram analisadas seis amostras de leite materno de mães de trigêmeos, 13 de gêmeos e 17 de filhos únicos, coletadas sob paramentação, conforme normas técnicas da Rede Brasileira de Bancos de Leite Humano. A análise foi realizada por meio do teste de crematócrito e os resultados submetidos à análise de variância de Kuskal-Wallis.

Resultados: A energia total do leite materno de mães de trigêmeos apresentou mediana de calorias maior $(651 \mathrm{kcal} / \mathrm{L})$, quando comparada à do leite de mães de filhos únicos $(560 \mathrm{kcal} / \mathrm{L})(p<0,05)$. O leite das mães de gêmeos e o de mães de filhos únicos não foram diferentes. Os trigêmeos apresentaram peso ao nascer menor (mediana 1344g) em relação aos filhos únicos (1975g) $(p<0,01)$. Não foi observada diferença estatística em relação à idade gestacional e à idade materna entre os três grupos de neonatos.

Conclusões: Pode-se concluir que o presente estudo, o primeiro na literatura sobre o tema, apontou para a presença de maior valor de energia total no leite de mães de recémnascidos trigemelares. Futuros estudos são necessários para elucidar os mecanismos envolvidos nesse achado.

Palavras-chave: banco de leite; leite materno; trigêmeos; gêmeos; recém-nascido.

\section{ABSTRACT}

Objective: To compare the total energy of the human milk of mothers of triplets, twins and single-children.

Methods: A cross-sectional and controlled study with mothers from the Human Milk Bank of Hospital Israelita Albert Einstein was performed. Six samples of triplet, 13 of twin and 17 of only-child mother's milk were analyzed. The milk samples were collected according to the Brazilian Human Milk Banks Network techniques and procedures and were analyzed by creamatocrit method. The results were compared by Kruskal-Wallis variance test.

Results: The total milk energy from triplets mothers was statistically higher (median - $651 \mathrm{kcal} / \mathrm{L}$ ) than singlechild mothers $(560 \mathrm{kcal} / \mathrm{L})(p<0.05)$. The milk of twin mothers and of single child mothers, regarding energy content, were similar. The triplet neonates presented lower birth weight (median-1344g) when compared to single neonates $(1975 \mathrm{~g})(p<0.01)$. There was no significant difference among the studied groups regarding maternal age and gestational age.

Conclusions: The present study, the first addressing this subject in literature, indicates a higher value of total energy in milk from triplets' mothers. However, further studies are necessary to clarify the mechanisms of this finding.

Key-words: milk bank; milk, human; triplets; twins; infant, newborn.
'Pós-graduanda do Programa em Nutrição e Ciências Aplicadas à Saúde da Universidade Federal de São Paulo (Unifesp), nutricionista e coordenadora do Serviço de Nutrição do Hospital Israelita Albert Einstein, São Paulo, SP, Brasil

${ }^{2}$ Professor titular do Departamento de Pediatria da Unifesp, São Paulo, SP, Brasil

3Professor titular da Disciplina de Saúde Coletiva da Universidade de Santo Amaro, São Paulo, SP, Brasil
Endereço para correspondência:

Ariane Nadólskis Severine

Avenida Albert Einstein, 627

CEP 05651-901 - São Paulo/SP

E-mail: ariane@einstein.br

Recebido em: 26/9/2007

Aprovado em: 11/3/2008 


\section{Introdução}

As vantagens oferecidas pelo aleitamento materno, em seus múltiplos aspectos, representam unanimidade no meio científico. A superioridade do leite humano, como alimento, agente protetor de infecções e modulador do crescimento e desenvolvimento do lactente, encontra-se amplamente descrita na literatura ${ }^{(1)}$.

A incidência de nascimento de gêmeos aumentou de 1 para $1,3 \%$ e de trigêmeos, de zero para $0,6 \%{ }^{(2)}$. Pesquisa realizada pela Society for Assisted Reproductive Technology mostrou que as taxas de sucesso globais médias de inseminação artificial em 1996, se comparadas aos dados de 1995, exibiram aumento real de 3,5\%. Estas taxas de sucesso variaram conforme o procedimento envolvido na fertilização in vitro (FIV), que representava dois terços dos procedimentos realizados em 1996, sendo bem sucedidas em 26\%. Na transferência intratubária de gametas (GIFT), em que o óvulo não fertilizado e o espermatozóide são introduzidos artificialmente na tuba uterina, observa-se sucesso de $29 \%{ }^{(3)}$. A maior eficácia das novas técnicas de reprodução assistida ${ }^{(4)}$ tem contribuído para incidência crescente de gestações múltiplas e de prematuridade ${ }^{(5)}$.

Com o aumento na freqüência de gestações múltiplas nos últimos anos, torna-se importante conhecer a composição do leite materno em tais situações, em relação à energia total, para assegurar a oferta energética adequada aos recémnascidos. Conhecer o conteúdo energético do leite de mães de recém-nascidos gemelares e trigemelares, comparado ao leite de mães de recém-nascidos únicos, é de grande relevância, pois não existe na literatura nenhum trabalho abordando este tema.

\section{Métodos}

Trata-se de estudo transversal e controlado, realizado com mães que utilizaram o banco de leite do Hospital Israelita Albert Einstein (HIAE), no período de maio de 2006 a maio de 2007. O trabalho foi aprovado pelos comitês de ética em pesquisa do HIAE e da Universidade Federal de São Paulo. O termo de consentimento livre e esclarecido não foi necessário para as mães usuárias dos bancos de leite, por se tratar de procedimento de controle incluído na rotina do setor e exigido pela Rede Brasileira de Bancos de Leite Humano $\left(\right.$ REDEBLH) ${ }^{(6)}$.

Participaram da presente pesquisa mães de trigêmeos, gêmeos e filhos únicos usuárias do banco de leite do HIAE e de uma maternidade privada. O banco de leite do HIAE não recebe doações de mães externas e trabalha somente com leite de mãe para filho. Entretanto, duas amostras foram coletadas em outro banco de leite de maternidade privada, com objetivo de aumentar o número de amostras de mães de trigêmeos. Tal maternidade, que apresenta características similares em relação ao perfil de usuárias e cujo serviço de banco de leite segue as mesmas normas do banco de leite do HIAE, cedeu duas amostras de leite envasadas e congeladas em tubo de ensaio contendo $2 \mathrm{~mL}$.

Devido ao fato de o nascimento de trigêmeos e gêmeos não ser previsível, optou-se por limitar o estudo pelo período de um ano. Dessa forma, o tamanho de amostra obtido foi constituído por seis mães de trigêmeos, 13 mães de gêmeos e 17 mães de filho único. O grupo de filhos únicos foi considerado grupo controle.

O leite materno foi coletado entre o primeiro e o $17^{\circ}$ dia após o parto e a coleta seguiu a norma técnica da REDEBLH (BLH-IFF/NT-16.04 - Ordenha: Procedimentos Higiênico-Sanitários) ${ }^{(6)}$. Conforme norma técnica, antes de iniciar o processo de coleta, as mães usuárias do banco de leite paramentam-se com touca, máscara e avental no setor de paramentação, higienizam as mãos e, imediatamente antes do início da coleta, utilizam gel alcoólico nas mãos.

A coleta foi realizada no banco de leite da maternidade no período da manhã. Para extrair o leite, utilizou-se bomba elétrica Medela ${ }^{\circledR}$ em ambas as mamas, até o esgotamento do volume de leite. Nos casos em que o volume foi superior a $120 \mathrm{~mL}$, empregaram-se dois frascos homogeneizados posteriormente, antes da retirada da alíquota a ser analisada.

As amostras foram separadas em tubo de ensaio com pipeta descartável, em alíquotas de $1 \mathrm{~mL}$, e armazenadas em freezer vertical domiciliar marca Brastemp, na temperatura de $-21^{\circ} \mathrm{C}$. Para realizar a técnica do crematócrito, as amostras foram descongeladas e aquecidas em banho-maria com temperatura controlada a $40^{\circ} \mathrm{C}$ por dez minutos.

Após o aquecimento e imediatamente antes do envasamento do leite no capilar de vidro, os tubos de ensaio com as alíquotas foram agitados individualmente durante dez segundos em vórtex. Em seguida, foram distribuídas em capilares de vidro, com circunferência interna de $1,0 \mathrm{~mm}$, circunferência externa de $1,5 \mathrm{~mm}$ e comprimento de $75 \mathrm{~mm}$ e posicionadas na microcentrífuga Fanem ${ }^{\circledR}$, com capacidade para 24 tubos. A rotação utilizada foi de 5.200 por 15 minutos. Para cada amostra, foram realizadas três medidas, considerando-se a média dos três resultados, conforme recomendação da REDEBLH ${ }^{(6)}$. 
Para leitura dos resultados, mediu-se a coluna total do leite e a coluna do creme, calculando-se a porcentagem de creme e, a seguir, o cálculo do valor energético, utilizando as seguintes fórmulas:

- Teor de creme $(\%)=$ coluna de creme $(\mathrm{em} \mathrm{mm}) \times 100 /$ coluna total de produto (em $\mathrm{mm}$ )

- Valor energético $(\mathrm{kcal} / \mathrm{L})=(\%$ creme x 66,8$)+290$

Para avaliação do crematócrito, foi utilizada a técnica de $\operatorname{Lucas}^{(7)}$.

Os dados da idade gestacional, idade materna e peso ao nascer foram coletados da ficha cadastral da mãe usuária do banco de leite.

$\mathrm{Na}$ análise dos resultados, foram aplicados os testes de Wilcoxon e de Kruskal-Wallis para comparar os três grupos em relação ao crematócrito, ao valor energético do leite, à idade gestacional e à idade materna ${ }^{(8)}$. Para comparar o peso de nascimento dos recém-nascidos entre os três grupos estudados, utilizou-se a análise de variância ${ }^{(9)}$. Fixou-se em 0,05 ou $5 \%$ o nível de rejeição da hipótese de nulidade.

\section{Resultados}

Os dados demográficos coletados apresentaram os seguintes resultados: a idade materna do grupo de trigemelares variou de 32 a 35 anos; no de gemelares, variou de 30 a 41 anos e, no grupo controle, de 28 a 46 anos. A idade gestacional variou no grupo de trigemelares de 29 a 33 semanas, nos gemelares variou de 27 a 33 semanas e, no controle, de 26 a 40 semanas. O peso de nascimento no grupo de trigemelares variou de 1145 a $2160 \mathrm{~g}$, nos gemelares de 980 a $2450 \mathrm{~g}$ e, no controle, de 670 a 3510 g. A mediana dos valores obtidos está apresentada na Tabela 1 .
Para saber se havia diferença no crematócrito em função da época após o parto, utilizou-se o teste de Wilcoxon, que mostrou não haver diferença na comparação em nenhuma das situações: trigêmeos, gêmeos e filho único ${ }^{(8)}$ (Tabela 2). A mediana do tempo de coleta do leite materno em relação ao parto para trigêmeos, gêmeos e filhos únicos foi de sete, oito e sete dias, respectivamente.

A energia total do leite de mães de trigêmeos foi significantemente maior que a do leite de mães de filhos únicos. $\mathrm{O}$ valor mediano da energia total do leite materno no grupo de trigêmeos foi $6511 \mathrm{kcal} / \mathrm{L}$ e $560 \mathrm{kcal} / \mathrm{L}$ para filho único (teste de Kruskal-Wallis; $p<0,05$ ). Não ocorreram diferenças quanto ao teor energético entre o leite de mães de trigêmeos e o de mães de gêmeos, nem entre o leite de mães de gêmeos em relação ao de mães de filho único.

\section{Discussão}

A técnica do crematócrito é uma alternativa que permite analisar e conhecer o conteúdo energético de forma precisa, simples, rápida e com baixo custo, de forma individual por amostras, sendo de utilidade indiscutível para adequada alimentação dos recém-nascidos em cuidados neonatais - principalmente para os que necessitam restrição hídrica e apresentam baixo ganho ponderal ${ }^{(10-12)}$. $\mathrm{O}$ leite materno, considerado como alimento ideal durante os primeiros seis meses de vida, deve ser conhecido em suas variações, principalmente para seu emprego em neonatos sob cuidados especiais ${ }^{(13-15)}$.

Os lipídios representam 50 a $60 \%$ do valor energético do leite materno e são os componentes mais variáveis ${ }^{(16,17)}$. O conteúdo energético do leite materno pode ser influenciado pela dieta materna ${ }^{(18-20)}$. O maior teor energético encontra-

Tabela 1 - Características gerais e conteúdo energético do leite materno expresso em mediana (valor minimo-máximo), segundo número de recém-nascidos em cada gestação.

\begin{tabular}{|c|c|c|c|c|}
\hline & $\begin{array}{c}\text { Trigêmeos } \\
6 \text { mães; } 18 \text { RN }\end{array}$ & $\begin{array}{c}\text { Gêmeos } \\
13 \text { mães; } 26 \text { RN }\end{array}$ & $\begin{array}{c}\text { Único } \\
17 \text { mães e RN }\end{array}$ & $p$ \\
\hline Idade mãe (anos) & $\begin{array}{c}32 \\
(32-35)\end{array}$ & $\begin{array}{c}35 \\
(30-41)\end{array}$ & $\begin{array}{c}32 \\
(28-46)\end{array}$ & $>0,05$ \\
\hline IG (semanas) & $\begin{array}{c}31 \\
(29-35)\end{array}$ & $\begin{array}{c}33 \\
(27-35)\end{array}$ & $\begin{array}{c}36 \\
(26-40)\end{array}$ & $>0,05$ \\
\hline Peso ao nascer (g) & $\begin{array}{c}1344 \\
(1145-2160)\end{array}$ & $\begin{array}{c}1356 \\
(980-2450)\end{array}$ & $\begin{array}{c}1975 \\
(670-3510)\end{array}$ & $<0,01$ \\
\hline Valor energético (kcal/L) & $\begin{array}{c}651 \\
(577-964)\end{array}$ & $\begin{array}{c}529 \\
(480-990)\end{array}$ & $\begin{array}{c}560 \\
(385-1068)\end{array}$ & $<0,05$ \\
\hline
\end{tabular}

IG: idade gestacional. 
Tabela 2 - Valor energético total (kcal/L) de cada amostra de leite materno de mães de trigêmeos, gêmeos e filho único, segundo o tempo de coleta após o parto

\begin{tabular}{|c|c|c|c|}
\hline & Coleta $\leq 5$ dias do parto & Coleta $>5$ dias do parto & Teste de Wilcoxon \\
\hline \multirow[t]{3}{*}{ Trigêmeos } & 682,78 & 958,00 & \\
\hline & 575,90 & 575,90 & \\
\hline & 671,42 & 910,29 & \\
\hline Mediana & 671,49 & 910,30 & $\mathrm{p}=0,40$ (NS) \\
\hline \multirow[t]{9}{*}{ Gêmeos } & 480,38 & 725,53 & \\
\hline & 483,05 & 480,38 & \\
\hline & 987,40 & 676,77 & \\
\hline & 542,50 & 483,05 & \\
\hline & & 584,58 & \\
\hline & & 671,42 & \\
\hline & & 671,42 & \\
\hline & & $1.005,42$ & \\
\hline & & 486,39 & \\
\hline Mediana & 512,77 & 671,42 & $\mathrm{p}=0,50$ (NS) \\
\hline \multirow[t]{14}{*}{ Único } & 384,85 & 480,38 & \\
\hline & 579,91 & 579,91 & \\
\hline & 575,90 & 388,19 & \\
\hline & & 579,91 & \\
\hline & & 480,38 & \\
\hline & & $1.075,56$ & \\
\hline & & 531,81 & \\
\hline & & 480,38 & \\
\hline & & 618,65 & \\
\hline & & 525,13 & \\
\hline & & 588,59 & \\
\hline & & 430,94 & \\
\hline & & 584,58 & \\
\hline & & 571,89 & \\
\hline Mediana & 575,90 & 551,85 & $\mathrm{p}=0,67$ (NS) \\
\hline
\end{tabular}

NS=não significante.

do no leite das mães de trigêmeos poderia estar associado ao maior cuidado em relação ao plano alimentar durante a gestação. A recomendação energética para suprir as necessidades da gestante gemelar é de aproximadamente $3.000 \mathrm{a}$ $4.000 \mathrm{kcal} / \mathrm{dia}$. Considera-se o acréscimo de 500 a $600 \mathrm{kcal} / \mathrm{dia}$ por feto ${ }^{(4,21)}$ às recomendações energéticas para a gestante.

A produção de lactogênio placentário é significantemente maior em gestações gemelares, comparadas àquelas de feto único ${ }^{(22)}$. É possível que tal produção seja ainda mais elevada em gestações de trigêmeos. Esse hormônio apresenta ação lactogênica e propicia a mobilização de ácidos graxos livres, o que poderia explicar o valor energético mais elevado no leite materno de mães de trigêmeos, comparativamente ao de gêmeos e filhos únicos ${ }^{(23)}$
Não se deve ignorar que a concentração de gordura do leite materno varia de mãe para mãe, independentemente do número de filhos daquele parto, assim como se observa variação no início e ao final da mamada e em períodos diferentes do dia ${ }^{(24)}$.

A literatura demonstra que mães com amamentação prolongada (período superior a um ano) apresentam aumento no conteúdo energético, comparadas àquelas com amamentação por período de dois a seis meses ${ }^{(25)}$. Desta forma, o período utilizado para coleta das amostras no presente estudo não ultrapassou 20 dias pós-parto, não interferindo nos resultados obtidos. A idade materna e a idade gestacional também não apresentaram diferença estatística significante entre os grupos estudados. 
Destaca-se, entre os fatores limitantes desse estudo, o pequeno número de amostras do grupo de trigêmeos e a ausência na literatura de pesquisas semelhantes, para comparação e discussão dos resultados encontrados.

Pode-se concluir que o presente trabalho, o primeiro na literatura sobre o tema, apontou para a presença de um maior valor de energia total do leite de mães de recémnascidos trigemelares. É possível que mães de gêmeos e trigêmeos sejam dotadas de um potencial maior para produção láctea do que mães de filho único devido aos aspectos relacionados à gravidez múltipla ${ }^{(26)}$. Futuros estudos são necessários para elucidar os mecanismos envolvidos nesse achado.

\section{Agradecimentos}

Agradecemos a todos que contribuíram direta e indiretamente para a realização deste trabalho.

\section{Referências bibliográficas}

1. Organização Mundial da Saúde (OMS). Proteção, promoção e apoio ao aleitamento materno: o papel especial dos serviços materno-infantis. Genebra: OMS; 1989.

2. Colletto GMDD. Twinning rate trend in a population sample from the city of São Paulo, Brazil. Genet Mol Biol 2003;26:245-8.

3. Hershlag A, Paine T, Cooper GW, Scholl GM, Rawlinson K, Kvapil G. Monozygotic twinning associated with mechanical assisted hatching. Fertil Steril 1999;71:144-6.

4. Colletto GM, Segre CA, Beiguelman B. Twinning rate in a sample from a Brazilian hospital with a high standard of reproductive care. Sao Paulo Med J 2001:119:216-9.

5. Werutsky NMA, Frangella VS, Pracanica D, Severine AN, Tonato C. Avaliação e recomendações nutricionais específicas para gestantes e puérperas gemelares. In: Silva SMCS, Mura JDP (editores). Tratado de alimentação, nutrição \& dietoterapia. São Paulo: Roca; 2007. p. 281-92.

6. Rede Brasileira de Bancos de Leite Humano. Manual técnico. Brasília: Ministério da Saúde; 2006. Available from: http://www.redeblh.fiocruz.br

7. Lucas A, Gibbs JA, Lyster RL, Baum JD. Creamatocrit: simple clinical technique for estimating fat concentration and energy value of human milk. Br Med J 1978;1:1018-20.

8. Siegel S (editor). Estatística não paramétrica. Rio de Janeiro: McgrawHill; 1975

9. Zar JH (editor). Biostatistical analysis. Upper Saddle River: Prentice Hall; 1999.

10. Smith L, Bickerton J, Pilcher G, D'Souza SW. Creamatocrit, carbon content, and energy value of pooled banked human milk: implications for feeding preterm infants. Early Hum Dev 1985;11:75-80.

11. Meier PP, Engstrom JL, Murtaugh MA, Vasan U, Meier WA, Schanler RJ. Mothers' milk feedings in the neonatal intensive care unit: accurancy of the creamatocrit technique. J Perinatol 2002;22:646-9.

12. Gregory K. Update on nutrition for preterm and full-term infants. J Obstet Gynecol Neonatal Nurs 2005;34:98-108.

13. Askin DF, Diehl-Jones WL. Improving on perfection: breast milk and breastmilk additives for preterm neonates. Newborn Infant Nurs Rev 2005;5:10-8.
14. Vinagre RD, Diniz EMA (editores). O leite humano e sua importância na nutrição do recém-nascido prematuro. São Paulo: Atheneu; 2001.

15. Calil VMLT, Leone CR, Ramos JLA. Composição nutricional do colostro de mães de recém-nascidos de termo adequados e pequenos para a idade gestacional. II - Composição nutricional do leite humano nos diversos estágios da lactação. Vantagens em relação ao leite de vaca. Pediatria (São Paulo) 1992;14:14-23.

16. Larnkjaer A, Schack-Nielsen L, Michaelsen KF. Fat content in human milk according to duration of lactation. Pediatrics 2006;117:988-9.

17. Almeida JAG (editor). Amamentação: um híbrido natureza-cultura. Rio de Janeiro: Fiocruz; 2002.

18. Mayans E, Martell M. Estimación del valor calórico de la leche materna mediante la técnica del crematocrito. Rev Med (Uruguay) 1994;10:160-4.

19. Lönnerdal B. Effects of maternal dietary intake on human milk composition. J Nutr 1986;116:499-513.

20. Harzer G, Dieterich I, Haug M. Effects of the diet on the composition of human milk. Ann Nutr Metab 1984;28:231-9.

21. Luke B. Nutrition in multiple gestations. Clin Perinatol 2005;32:403-29.

22. Kazer RR, Cheng ER, Unterman TG, Glick RP. Maternal plasma concentration of insulin-like growth factor-I (IGF-I) and human placental lactogen (hPL) in twin pregnancies. Acta Genet Med Gemellol (Roma) 1991;40:383-7.

23. Forsyth IA. Variation among species in the endocrine control of mammary growth and function: the roles of prolactin, growth hormone, and placental lactogen. J Dairy Sci 1986;69:886-903.

24. Aksit S, Ozkayin N, Caglayan S. Effect of sucking characteristics on breast milk creamatocrit. Paediatr Perinat Epidemiol 2002;16:355-60.

25. Mandel D, Lubetzky R, Dollberg S, Barak S, Mimouni FB. Fat and energy contents of expressed human breast milk in prolonged lactation. Pediatrics 2005;116:e432-5.

26. Berlin CM. "Exclusive" breastfeeding of quadruplets. Breastfeed Med 2007;2:125-6. 